\title{
Severe Central Sleep Apnea/Hypopnea Syndrome Mimicking Rapid Eye Movement Sleep Behavior Disorder
}

\author{
Joon Mo Koo, MD, Su-Hyun Han, MD, Sang-Ahm Lee, MD \\ Department of Neurology, Asan Medical Center, University of Ulsan College of Medicine, Seoul, Korea
}

\begin{abstract}
Rapid eye movement sleep behavior disorder (RBD) is a sleep disorder characterized by loss of muscle atonia during rapid eye movement (REM) sleep associated with dream enactment. However, these complex behaviors can also occur in other sleep disorders, a clinical situation termed as "pseudo-RBD". Anecdotal reports of obstructive sleep apnea syndrome simulating the clinical features of RBD have been reported. It has been suggested that "pseudo-RBD" is a form of a confusional arousal caused by a combination of severe oxyhemoglobin desaturations and other unknown factors. However, there have been no previous reports of RBD-like behaviors associated with central sleep apnea. Here, we describe two patients who reported complex motor and vocal behavior mimicking RBD, but REM sleep without atonia was not observed in video-polysomnography (VPSG). Furthermore, VPSG demonstrated severe central sleep apnea-hypopnea inducing abnormal motor and vocal behaviors.

Sleep Med Res 2015;6(2):77-80
\end{abstract}

Key Words Central sleep apnea/hypopnea, Rapid eye movement sleep behavior disorder, Pseudo-rapid eye movement sleep behavior disorder.

Received: December 2, 2014

Revised: February 16, 2015

Accepted: April 6, 2015

Correspondence

Sang-Ahm Lee, MD

Department of Neurology,

Asan Medical Center,

University of Ulsan College of Medicine,

88 Olympic-ro 43-gil, Songpa-gu,

Seoul 05505, Korea

Tel $+82-2-3010-3445$

Fax +82-2-474-4691

E-mail_salee@amc.seoul.kr

\section{INTRODUCTION}

Rapid eye movement sleep behavior disorder (RBD) is a sleep disorder characterized by loss of muscle atonia during rapid eye movement (REM) sleep associated with dream enactment. ${ }^{1}$ RBD may be idiopathic or associated with neurodegenerative diseases. ${ }^{1}$ Patients with RBD display complex motor behaviors such as gesturing, falling, talking, and shouting that are associated with unpleasant dreams. However, these abnormal sleep behaviors can also occur in other sleep disorders such as obstructive sleep apnea syndrome (OSAS). ${ }^{2}$ This clinical situation has been termed "pseudo-RBD". Iranzo and Santamaría ${ }^{2}$ have identified patients who presented with abnormal sleep behaviors and unpleasant dreams in addition to OSAS. "Pseudo-RBD" has been reported in five cases of severe OSAS, they presented violent behaviors during OSAS-induced arousals from REM sleep. ${ }^{3}$ Other anecdotal reports of OSAS simulating the clinical features of RBD also have been reported. ${ }^{4,5}$

Nevertheless, there have been no previous reports of pseudo-RBD associated with central sleep apnea-hypopnea (CSAH) syndrome. Here, we describe two patients who display complex behaviors mimicking RBD, but REM sleep without atonia was not observed in videopolysomnography (VPSG). Furthermore, VPSG demonstrated abnormal motor behaviors related to arousals induced by severe CSAH.

\section{CASE REPORT}

\section{Patient 1}

An 82 year old woman came into our hospital with sleep disturbance. She had abnormal behaviors during sleep, such as talking, singing and pounding on the wall, and also had unpleas- 
ant dreams and daytime hypersomnolence. She had been taking antihypertensive medicine for 20 years and there was a history of intracranial hemorrhage on the right basal ganglia 15 years ago. Recently, she was diagnosed with diagnostic heart failure by a cardiologist. VPSG was done. Sleep architecture showed an increased amount of N1, and decreased N3 and R sleep. Sleep latency was 0.5 minutes. The apnea-hypopnea index (AHI) was $52.1 / \mathrm{h}$, total arousal index was $23.8 / \mathrm{h}$ and apnea-hyponea arousal index was $16.1 / \mathrm{h}$. The lowest oxygen saturation was $88 \%$. She showed a waxing and waning pattern of ventilation, which is suggestive of Cheyne-Stokes respiration (Fig. 1). Talking and singing behaviors were recorded related to arousals during a hyperventilation period in $\mathrm{N} 1$, and no event including REM sleep without atonia (RSWA) was detected during R sleep (Fig. 1). Therefore, we diagnosed this patient as pseudo-RBD related to CSAH.
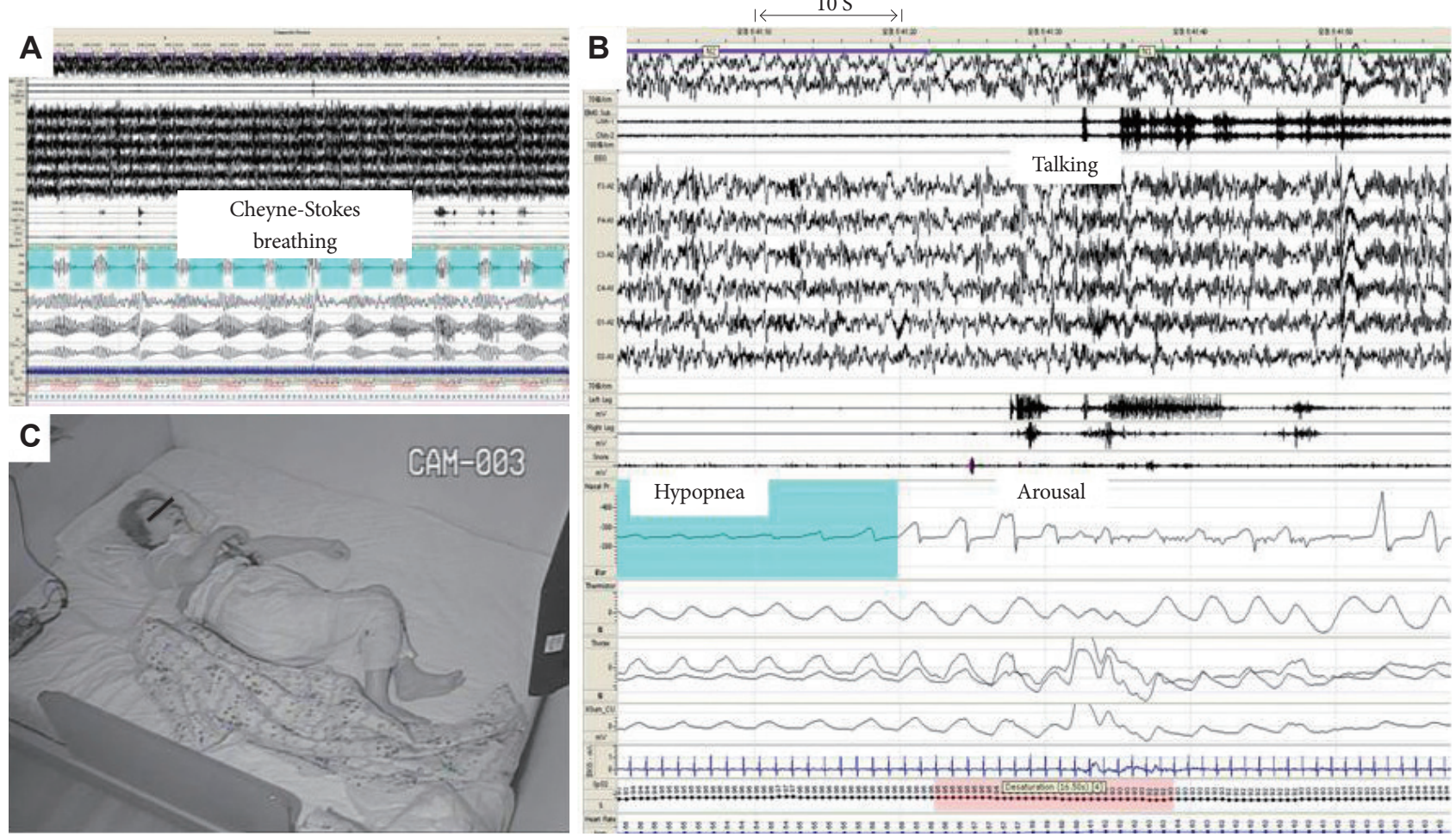

Fig. 1. A and B: Polysomnographic recording of patient 1 shows the evidence of typical Cheyne-Stokes respiration. C: RBD-like symptom (talking) was recorded in N1 sleep related to arousal. RBD: rapid eye movement sleep behavior disorder.
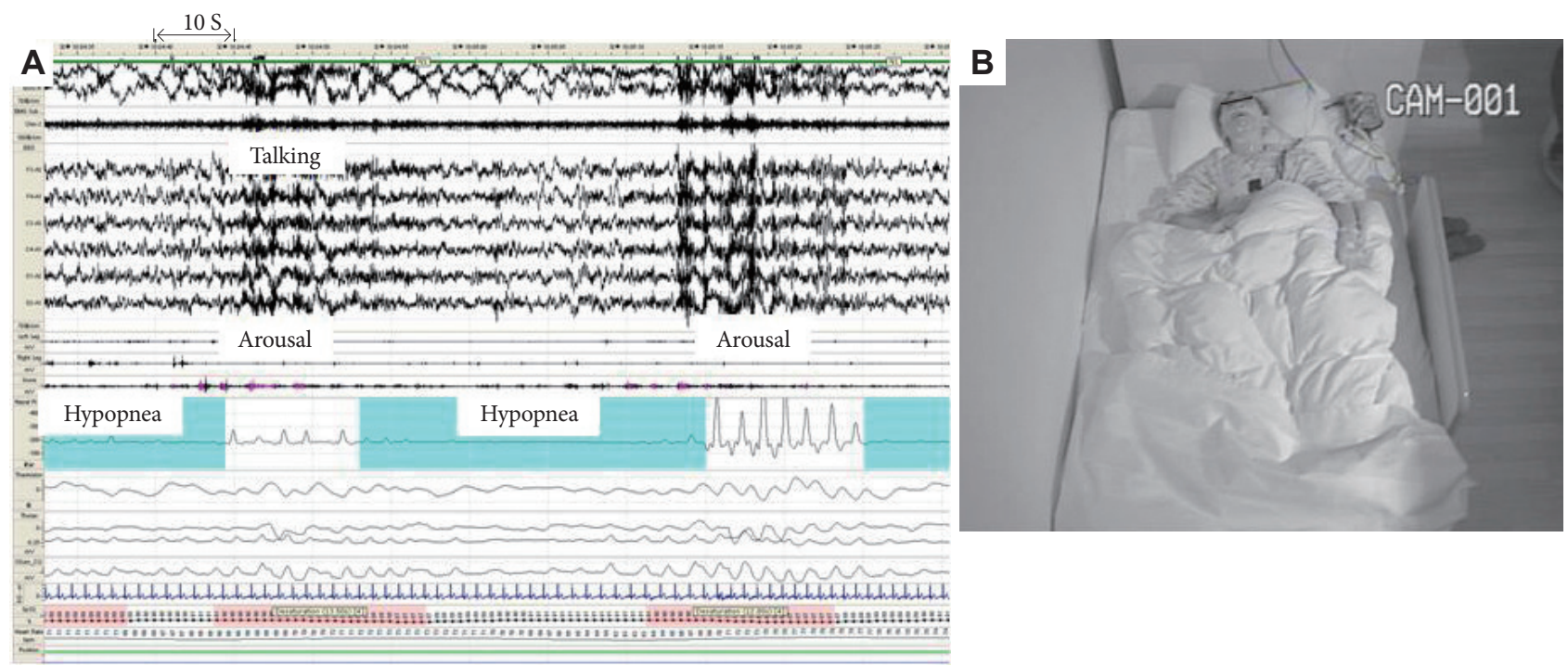

Fig. 2. A: Polysomnographic recording of patient 2 shows a periodic breathing pattern. B: Talking and body movements were recorded in N1 sleep related to arousal. 


\section{Patient 2}

A 60 year old man had suffered from progressive motor weakness for a year. His symptoms started with weakness in both legs, and 6 months later muscle wasting was observed, as well as dyspnea on minimal movement. The findings of an electromyography suggested anterior horn cell disease. He also had newly-developed abnormal sleep behavior such as gesturing or talking, frequent awakening during sleep, and daytime fatigue since 4 months. In VPSG, sleep architecture showed an increased amount of N1, and decreased N2, and no N3, R sleep. Sleep latency was 3.0 minutes. AHI was $120 / \mathrm{h}$, total arousal index was 119.6/h and apnea-hyponea arousal index was 115.0/h. The lowest oxygen saturation was $82 \%$. He showed a periodic breathing pattern (Fig. 2). Talking and body movements were recorded in N1 sleep at the end of a hypopnea period accompanied with arousals, and RSWA was not detected (Fig. 2). Therefore, central sleep apnea and accompanied pseudo-RBD was diagnosed.

\section{DISCUSSION}

Here, we report two patients who presented with complex sleep behaviors in addition to CSAH syndrome. Their clinical presentations were similar to RBD, and initially we thought they may have had RBD. However, in VPSG, we identified CSAH and abnormal behaviors occurring in respiratory effort-related arousals, not in the REM sleep period.

Previous studies ${ }^{2-4}$ have indicated that, in patients with OSAS, "pseudo-RBD" is only seen in those patients with a high $\mathrm{AHI}$ and severe oxyhemoglobin desaturations. It can be postulated that in some patients with severe OSAS syndrome, "pseudo-RBD" is a form of confusional arousal caused by a combination of severe oxyhemoglobin desaturations and other unknown factors. ${ }^{2}$ However, the lowest $\mathrm{O} 2$ saturations were $88 \%$ and $82 \%$ in our patients, which are not severe compared with that reported in the previous study ${ }^{2}$ (mean minimal oxyhemoglobin saturation $57.2 \pm 18.8 \%$ ). It is due to the fact that arousals are associated more with hyperventilation than with severe desaturation in non-hypercapnia CSAH patients like ours.

Central sleep apnea-hypopnea syndrome is characterized by a lack of drive to breathe during sleep, resulting in insufficient ventilation and compromised gas exchange, repetitively. ${ }^{6}$ CSAH can be broadly classified into hypercapnic and non-hypercapnic. ${ }^{6}$ In our two patients, we could identify non-hypercapnic CSAH such as Cheyne-Stokes breathing and a periodic breathing pattern, which is quite different from hypercapnic CSAH that diminishes ventilatory output. ${ }^{6}$ Non-hypercapnic $\mathrm{CSAH}$ promotes unstable breathing such as a high ventilatory drive, and vulnerability of the arousal and accompanying hyperventilation. ${ }^{6}$ Therefore, it is speculated that the patient with non-hypercapnic CSAH can experience frequent arousals com- pared to hypercapnic CSAH, and is more likely to have "pseudo-RBD" due to these frequent arousals. Additionally, arousals typically occur in the mid-cycle of Cheyne-Stokes respiration, at the peak of ventilator effort, ${ }^{7}$ and at the termination of central apnea in periodic breathing. ${ }^{6}$ We can also see those patterns of arousals in our patient's polysomnography (PSG). Therefore, it is postulated that non-hypercapnic CSAH, as our patients, rather than hypercapnic CSAH, can lead to "pseudo-RBD" due to frequent arousals.

To distinguish RBD from CSAH is important because these two diseases have different clinical implications. CSAH is caused by brainstem structures or various neuromuscular disorders, and its complications are secondary polycythemia, pulmonary hypertension, and cor pulmonale. ${ }^{6}$ Additionally, Cheyne-Stokes respiration is most commonly observed in patients with congestive heart failure and left ventricular systolic dysfunction. ${ }^{6}$ Regarding RBD, the diagnosis in a patient with no evidence of neurodegenerative disease must be clearly identified because idiopathic RBD can frequently be a prodromal symptom of neurodegenerative diseases.

Pseudo-RBD may also occur in other conditions such as nocturnal seizures, ${ }^{8}$ night terrors ${ }^{4}$ and nocturnal wanderings. ${ }^{9}$ In these situations, only VPSG can exclude RBD and detect these other disorders. Therefore, VPSG is essential to find concomitant sleep disorders, to distinguish true RBD and pseudo-RBD for patients suffering abnormal sleep behaviors resembling RBD.

In conclusion, we are the first to report two patients with pseudo-RBD associated with CSAH. Unlike that in OSAS, pseudo-RBD in CSAH may not present severe oxyhemoglobin desaturation because arousals in CSAH are usually related to hyperventilation. And among the two types of CSAH, non-hypercapnic CSAH rather than hypercapnic CSAH can result in pseudo-RBD due to frequent arousal during sleep. PseudoRBD may occur in various sleep disorders including CSAH and OSAS, and in these situations, PSG can exclude RBD and detect these other disorders.

\section{Conflicts of Interest}

The authors have no financial conflicts of interest.

\section{REFERENCES}

1. Schenck CH, Mahowald MW. REM sleep behavior disorder: clinical, developmental, and neuroscience perspectives 16 years after its formal identification in SLEEP. Sleep 2002;25:120-38.

2. Iranzo A, Santamaría J. Severe obstructive sleep apnea/hypopnea mimicking REM sleep behavior disorder. Sleep 2005;28:203-6.

3. Nalamalapu U, Goldberg R, DiPhillipo M, Fry JM. Behaviors simulating REM behavior disorder in patients with severe obstructive sleep apnea. Sleep Res 1996;25:311.

4. Schenck CH, Milner DM, Hurwitz TD, Bundlie SR, Mahowald MW. A polysomnographic and clinical report on sleep-related injury in 100 adult patients. Am J Psychiatry 1989;146:1166-73.

5. Boeve BF, Silber MH, Ferman TJ, Lucas JA, Parisi JE. Association of REM sleep behavior disorder and neurodegenerative disease may re- 
flect an underlying synucleinopathy. Mov Disord 2001;16:622-30.

6. Eckert DJ, Jordan AS, Merchia P, Malhotra A. Central sleep apnea: Pathophysiology and treatment. Chest 2007;131:595-607.

7. Trinder J, Merson R, Rosenberg JI, Fitzgerald F, Kleiman J, Douglas Bradley T. Pathophysiological interactions of ventilation, arousals, and blood pressure oscillations during cheyne-stokes respiration in patients with heart failure. Am J Respir Crit Care Med 2000;162(3 Pt
1):808-13.

8. Boller F, Wright DG, Cavalieri R, Mitsumoto H. Paroxysmal "nightmares". Sequel of a stroke responsive to diphenylhydantoin. Neurology 1975;25:1026-8

9. Pedley TA, Guilleminault C. Episodic nocturnal wanderings responsive to anticonvulsant drug therapy. Ann Neurol 1977;2:30-5. 